\title{
Temperature dependence of friction under cryogenic conditions in vacuum
}

\author{
J. C. Burton, P. Taborek* and J. E. Rutledge \\ Department of Physics and Astronomy, University of California, 4129 Fredrick Reines Hall, Irvine, CA 92697, USA
}

Received 12 June 2006; accepted 21 July 2006; published online 26 August 2006

\begin{abstract}
We have constructed a sliding block cryotribometer designed to measure friction at cryogenic temperatures and UHV conditions. An optical cryostat and high speed video was used to monitor the motion of the block. The temperature range of the measurements was 4-460 K. Both the static and dynamic friction coefficients were determined for a steel ball on stainless steel, sapphire, and PTFE (Teflon) plates, and for a sapphire ball on a sapphire plate. For all material combinations, no systematic variation of friction with temperature was observed in the temperature range 4-200 K.
\end{abstract}

KEY WORDS: cryotribology, friction test methods, static friction, PTFE, unlubricated wear

\section{Introduction}

Many thermodynamic and transport properties of solids depend dramatically on the temperature. Properties determined by phonons such as the heat capacity and thermal diffusivity often differ by orders of magnitude above and below the Debye temperature. In both metals and semiconductors, the electrical resistivity can have a strong temperature dependence, particularly in the cryogenic regime. Since a fundamental description of friction between solids involves electronic and phonon coupling between the interfaces [1], it is of interest to investigate whether the temperature dependence of the scattering properties of the elementary excitations is reflected in observable variation of the friction coefficients with temperature. More macroscopic theories [2] of frictional phenomena use models based on effects such as local melting of asperities, viscoelastic effects, plastic flow, and tribochemistry [3]. The rates of these processes have a strong Arrhenius-type temperature dependence, with the local temperature determined by a balance of heat generation at the surface and heat flow away from the sliding surface. At sufficiently low temperatures, the rate of thermally activated processes becomes vanishingly small, and processes which are important at room temperature can be effectively turned off. Another theoretical approach leads to a frictional force with an additive term that is proportional to the temperature $T$ and to the logarithm of the sliding velocity $[4,5]$. Thus, from both the fundamental and the macroscopic phenomenological point of view, temperature might be

\footnotetext{
*To whom correspondence should be addressed. E-mail: ptaborek@uci.edu
}

expected to play an important role in sliding friction, particularly in the cryogenic regime. Despite this theoretical expectation, there have been very few experimental investigations of friction at low temperatures, and the studies which do exist often involve surfaces in contact with gases or cryogenic fluids [6-10] which complicates the interpretation of the data in terms of interfacial processes. Other experiments have used AFM techniques to study friction at single asperities on well characterized single crystal surfaces in vacuum [11].

The purpose of the measurements described here is to provide a survey of friction behavior over a wide range of temperature for materials of engineering importance. The materials we have chosen, stainless steel, Teflon (PTFE), and sapphire, are representative of metals, polymers, and crystalline insulators whose electronic and mechanical properties span a wide range. Friction measurements on macroscopic samples in vacuum at cryogenic temperatures pose significant experimental challenges and require special purpose tribometers. We have chosen a conceptually simple but rather unusual technique which involves monitoring the motion of a block sliding down an inclined plane using high speed video. The video frames are analyzed to yield the acceleration and force on the sliding block, from which the sliding friction coefficient can be determined. The static friction coefficient is obtained from the critical angle of inclination required to initiate sliding. Contrary to our initial expectations, our results for the friction coefficients show very little temperature dependence in the range 4-200 K. Reproducible thermal effects were observed in the temperature range 200-400 K, but wear was found to be a more important effect than temperature. 


\section{Experimental methods}

Our tribometer consists of a $2 \mathrm{~cm} \times 1.27 \mathrm{~cm} \times 1.27 \mathrm{~cm}$ copper block which slides in a vee-shaped track approximately $10 \mathrm{~cm}$ long. Two of the faces of the copper block slider have three $3.175 \mathrm{~mm}$ (1/8 inch) diameter balls made from bearing steel attached so that approximately half of the ball is exposed; these hemispherical contact points support the load and define the tribological contact points. In the limit of zero friction, five contact points determine the orientation of the slider in the track, so six contact points over constrain the system. The friction coefficients we measure, however, depend only on the sum of the normal forces exerted by all of the contact points, and not on the details of the force distribution. Most of our measurements were done with steel balls, but one experimental run was done with sapphire balls (purchased from Small Parts). The balls make contact with two rectangular plates mounted at 90 degrees which form the vee. The main body of the track holder is made of OFHC copper for thermal management reasons. The tracks are mounted in machined recesses in the copper body of the track holder and are replaceable; we used highly polished stainless steel (McMasterCarr 304 ss mirror-like finish), sapphire (Crystal Systems, 80-50 optical polish, unknown crystallographic orientation) and PTFE coated steel (Dupont 851-214Green PTFE coating 73 microns thick). A schematic diagram of the slider is shown in figure 1.

The track holder is mounted on a frame which hangs from the upper surface of a $4 \mathrm{~K}$ platform in a cryostat. The holder is mounted on bushings which allow it to rotate around a horizontal axis. Its angular position is controlled by a wire attached to a linear feedthru at the top of the cryostat. The entire tribometer is completely enclosed by a copper radiation shield which is attached to the $4 \mathrm{~K}$ platform. Optical access is provided by two windows of infrared absorbing glass(Schott KG-1). The $4 \mathrm{~K}$ shield is inside a $77 \mathrm{~K}$ radiation shield which also has infrared absorbing windows. The cryostat is mounted in a conventional stainless steel vacuum tank $46 \mathrm{~cm}$ in diameter with quartz windows in Conflat ports, as shown in figure 2 . The design of the cryostat is similar to other optical cryostats used previously in our laboratory [12-14].

While the apparatus is at room temperature, it is pumped out to a pressure of $10^{-6}$ Torr. Once the radiation shields of the cryostat reach their final temperatures of approximately 4 and $77 \mathrm{~K}$, respectively, the region surrounding the tribometer is effectively cryopumped by the shields, and the pressure presumably drops to very low values. The temperature of the tribometer is monitored and controlled independently of the shields using a heater and thermometer attached to the copper body of the vee-shaped track. Because of the point-like contacts of the balls, the sliding block makes poor thermal contact with the track; if this were the only

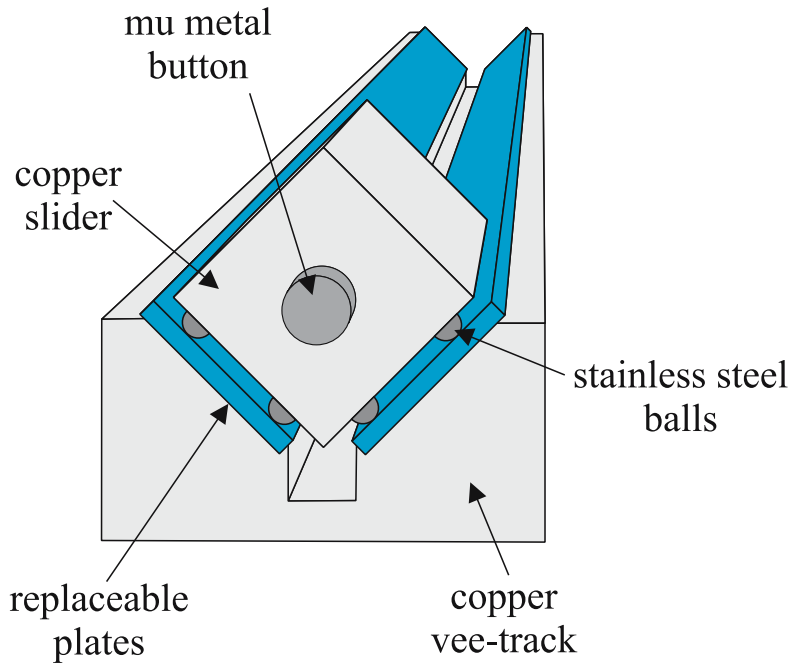

Figure 1. Schematic diagram showing copper slider supported on ball bearings which slides on replaceable plates that form a 90 degree vee track. The mu metal button is used in conjunction with the solenoid to provide thermal contact to the copper slider and to control the release of the slider at high inclination angles.

thermal connection to the track, the temperature of the block would be impossible to control. To enhance the thermal contact, a high magnetic permeability disk of mu metal is attached at one end of the block which can be attracted to the mu metal core of a solenoid at the end of the track. The solenoid can hold the block at the end of the track even when the track is tilted at a large angle. When the current through the coil is switched off, the block can slide freely.

Once the block and track have reached a thermal steady state, the static and kinetic friction coefficients $\mu_{\mathrm{s}}$

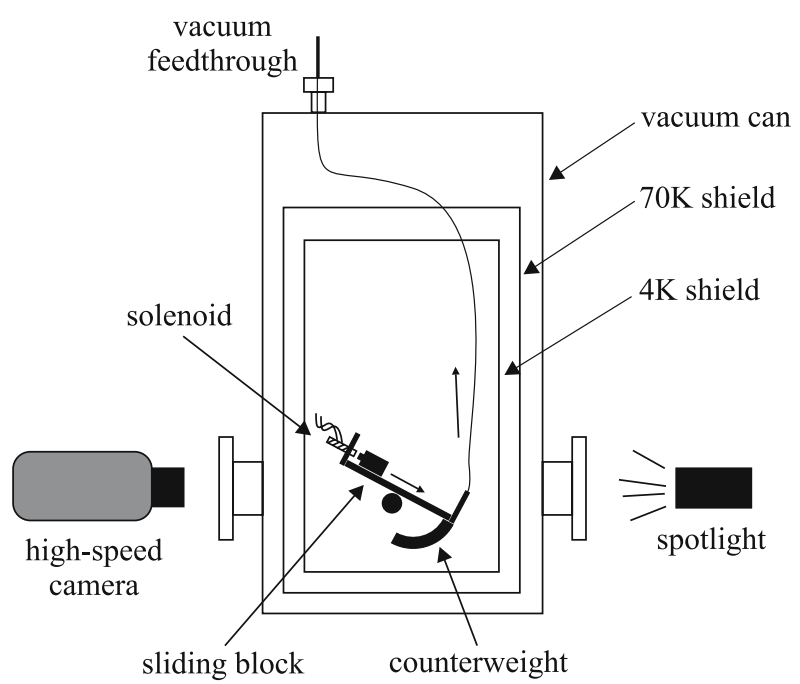

Figure 2. Schematic diagram of the cryostat and sliding block tribometer. The vee-shaped track can be rotated around a horizontal axis by pulling against a counterweight with a thin flexible wire attached to a vacuum feedthrough. In the actual experiment, the camera and the light source were collinear with the axis of rotation. 
and $\mu_{\mathrm{k}}$ can be determined by using the video camera to monitor the motion of the block. The forces on the block are illustrated in figure $3 . \mu_{\mathrm{s}}$ is determined by slowly increasing the angle of inclination $\theta$ until motion begins at $\theta=\theta_{0} . \theta_{0}$ is measured from the video image. At $\theta_{0}$, the component of the gravitational force pulling the block down the track $m g \sin \theta_{0}$ and the frictional force retarding the motion $\sqrt{2} \mu_{\mathrm{s}} m g \cos \theta_{0}$ are in balance, so the static coefficient of friction is

$$
\mu_{\mathrm{s}}=\frac{\tan \theta_{0}}{\sqrt{2}}
$$

The coefficient of kinetic friction $\mu_{\mathrm{k}}$ is determined by analyzing the video frames of the motion of the block as it slides down the track. To avoid the stick-slip motion characteristic of sliding near the critical angle, sliding friction measurements were performed by clamping the block at the end of the track using the solenoid, inclining the track at an angle approximately $10^{\circ}$ higher than the critical angle, and then releasing the block. The block is illuminated from behind using a conventional photographic lamp. The infrared filters on the shields minimize the heat load on the tribometer; the temperature is observed to rise approximately $0.5 \mathrm{~K}$ when the lamp is turned on near $4 \mathrm{~K}$, and less than $50 \mathrm{mK}$ at higher temperatures. We used a Phantom V7.2 camera which produces digital images with $512 \times 384$ pixels at a typical frame rate of $1000 \mathrm{fps}$; the spatial resolution of the image using our optics is 250 microns per pixel. The camera was carefully mounted in the horizontal position to within $\pm 0.1^{\circ}$ using a digital level. A software package written in Mathematica using standard convolution techniques tracks the position of a corner of the block from one frame to the next and produces a list of positions as a function of time. The acceleration is determined by constructing a least squares fit of a quadratic polynomial to the trajectory; a typical example is shown in figure 4.

The high quality of the quadratic fit indicates that, to a good approximation, the block slides with constant

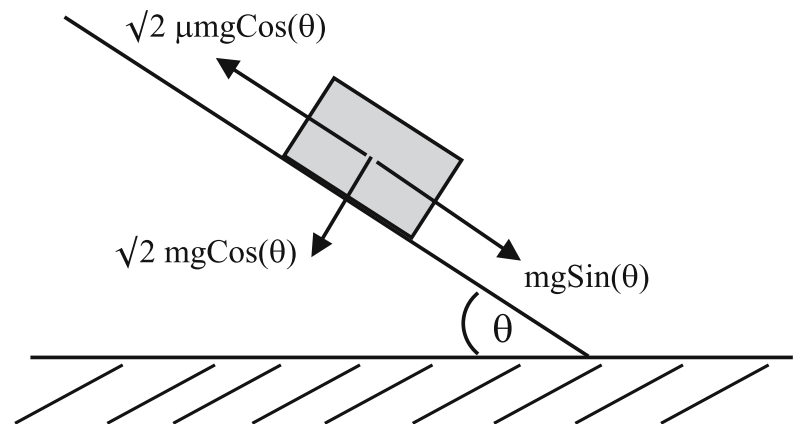

Figure 3. Free body diagram showing the total forces on the sliding block. The factors of $\sqrt{2}$ are due to fact that the load bearing faces of the block are inclined at an angle of $45^{\circ}$, as shown in figure 1 . The total normal force is made up of the sum of the normal force exerted by each face of the vee: $m g \cos (\theta) \sqrt{2}+m g \cos (\theta) \sqrt{2}=\sqrt{2} m g \cos (\theta)$.

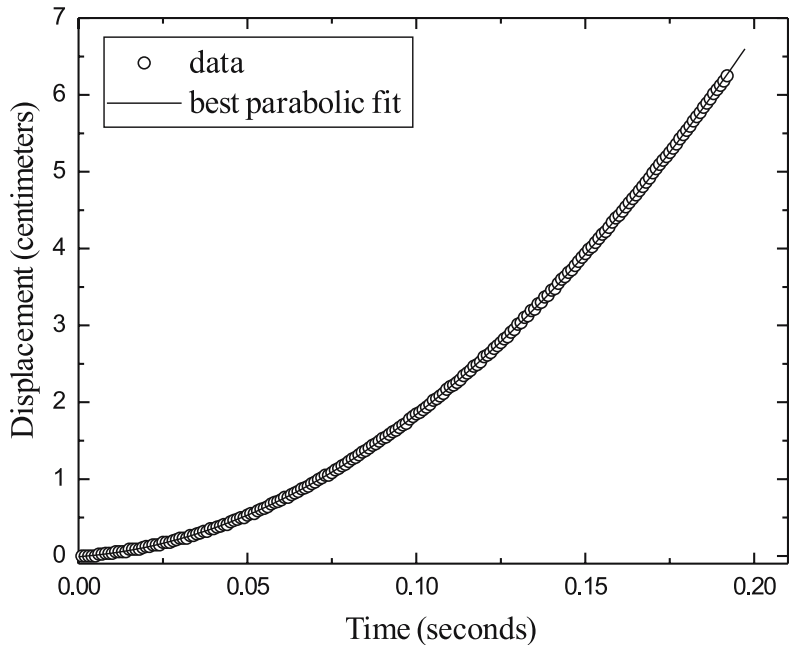

Figure 4. Displacement of the block parallel to the track as a function of time. Open circles are the position determined from the video frames. The solid curve is a least squares fit of a quadratic polynomial. The coefficient of the quadratic term is directly related to the acceleration.

acceleration $a$ that can be determined from the second derivative of the the best fit polynomial. It also implies that if there is a term in the frictional force proportional to the logarithm of the velocity, it must be very small for the conditions of our apparatus. The coefficient of kinetic friction is determined from

$$
\mu_{k}=\frac{\tan \theta}{\sqrt{2}}-\frac{a}{g \sqrt{2} \cos \theta}
$$

The statistical uncertainty in the value of $\mu_{\mathrm{k}}$ obtained from data like those shown in figure 4 using Equation 2 is remarkably small and is of the order of $10^{-3}$. Uncertainty in the angle introduces a systematic error of roughly the same amount.

\section{Friction as a function of temperature}

Friction measurements as described above were performed using steel balls on three types of tracks: stainless steel, polished sapphire, and PTFE coated steel shown in figures 5, 6 and 8, respectively. One run was done using sapphire balls on a sapphire plate as shown in figure 7. Each experimental run was started with a new set of balls in the block and a fresh sample of the track material. We found that the run-in history of the samples strongly affected the friction. Each set of measurements is denoted in the figures by colors and symbols labeled "cooldown1", "cooldown2" , "warmup1", "warmup2", etc., which describe the thermal cycling as well as the wear history of the sample. All of the experimental runs were started with the tribometer and the cryostat shields near room temperature and the pressure at approximately $10^{-6}$ Torr. For the hard track materials, steel and sapphire, this first friction 

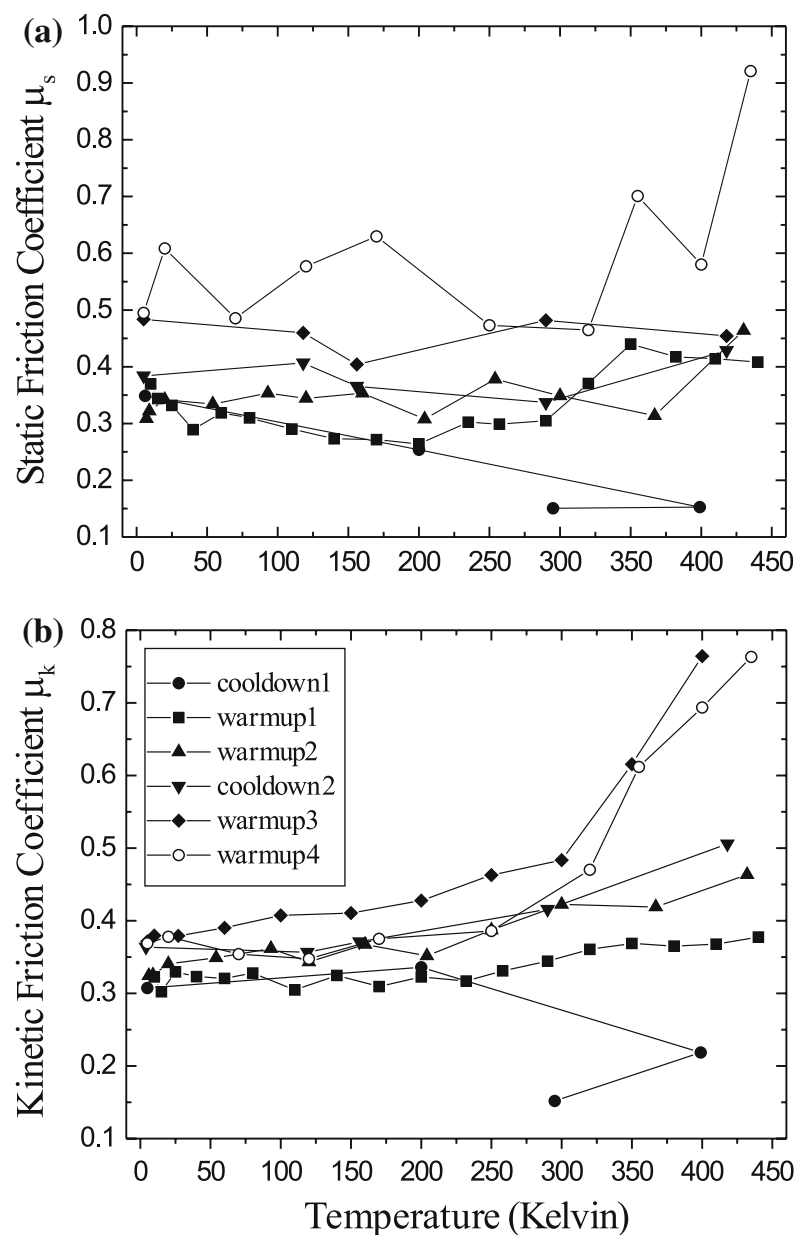

Figure 5. Friction coefficients of stainless steel on stainless steel as a function of temperature. The symbols indicate whether the data was taken on heating or cooling, and the line segments connecting the data points indicate the order in which they were measured. (a) static friction, (b) sliding friction.

measurement gave the lowest values we observed throughout the sequence of measurements. Next, the temperature of the cryostat shields was lowered to their final values and simultaneously, the temperature of the tribometer was raised to approximately $400 \mathrm{~K}$. This presumably has the effect of outgassing the tribometer surfaces and cryopumping the apparatus. The temperature of the tribometer was then ramped several times from approximately $400 \mathrm{~K}$ down to $4 \mathrm{~K}$ and back again, and friction measurements were obtained at intervals of approximately $20 \mathrm{~K}$. A noteworthy feature of the data is that although the statistical error in $\mu$ obtained from a single slide down the track was very small, the fluctuations in $\mu$ obtained from a series of measurements at the same or similar temperatures was much larger.

Our results for friction of steel on steel as a function of temperature are shown in figure 5. The Hertzian contact stress is computed assuming 5 balls are in contact, and is approximately $232 \mathrm{MPa}$. At room temperature, the kinetic friction coefficient $\mu_{\mathrm{k}}$ is initially 0.15 , but during the first cooling cycle, it rises to approximately 0.3 . We believe most of this change can be attributed to the rapid wear that occurs in high vacuum $[15,16] . \mu_{\mathrm{k}}$ increases very gradually over several cooling and heating cycles, which is also presumably due to wear. The only substantial temperature dependence evolves after considerable wear, when the samples show a reproducible and reversible increase from $\mu_{\mathrm{k}} \asymp 0.4$ to $\mu_{\mathrm{k}} \asymp 0.8$ in the range $300 K<T<450 K$. The static friction coefficient $\mu_{\mathrm{s}}$ qualitatively follows the same pattern.

The friction data for steel on sapphire is shown in figure 6 . The Hertzian contact stress is approximately $276 \mathrm{MPa}$. The initial values of $\mu_{\mathrm{k}}$ are approximately 0.15 , but as the sample wears in, $\mu_{\mathrm{k}}$ increases to the range $0.4-0.45$. There is very little temperature dependence, particularly below $250 \mathrm{~K}$. Above $250 \mathrm{~K} \mu_{\mathrm{k}}$ decreases slightly with temperature. The static friction coefficient shows qualitatively the same behavior. The friction of sapphire on sapphire shown in figure 7 also follows the same pattern: initially, the friction is very
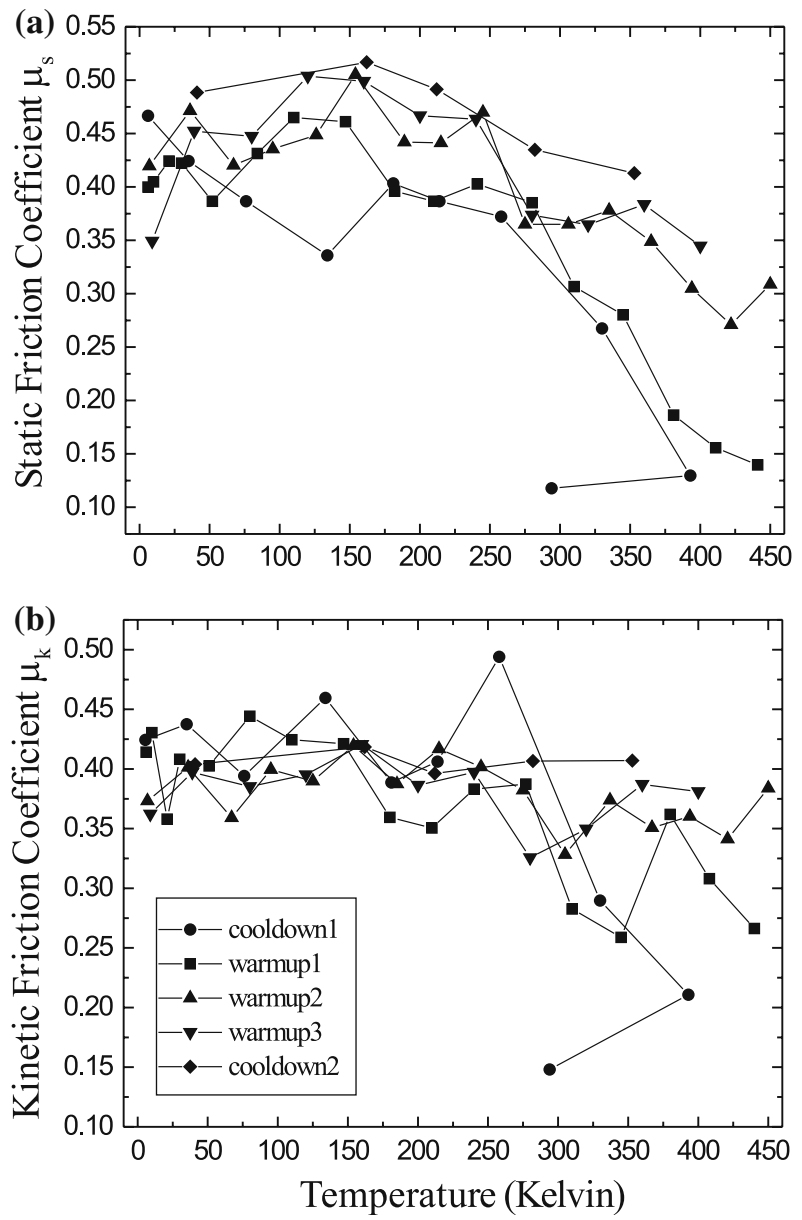

Figure 6. Friction coefficients of stainless steel on sapphire as a function of temperature. The symbols indicate whether the data was taken on heating or cooling, and the line segments connecting the data points indicate the order in which they were measured. (a) static friction, (b) sliding friction. 

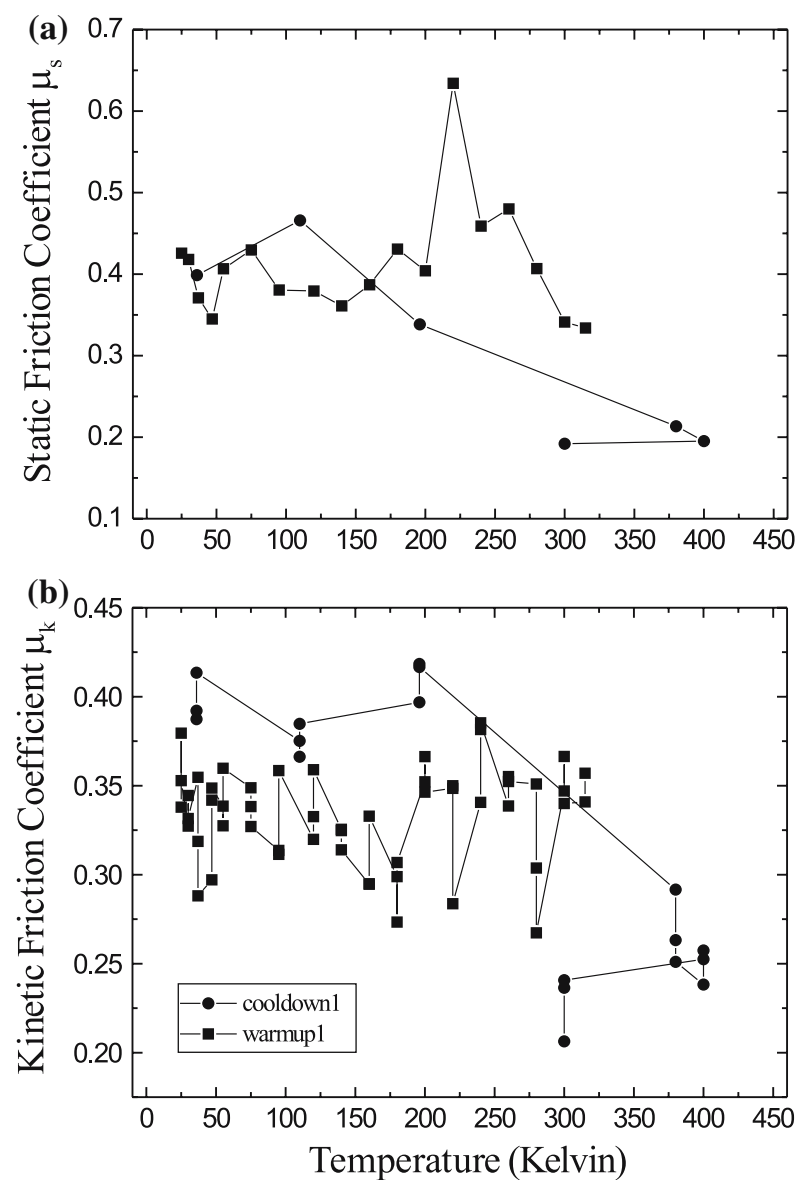

Figure 7. Friction coefficients of sapphire on sapphire as a function of temperature. The symbols indicate whether the data was taken on heating or cooling, and the line segments connecting the data points indicate the order in which they were measured. (a) static friction, (b) sliding friction.

low, but the wear caused by a few sliding cycles increases $\mu_{\mathrm{k}}$ to approximately 0.4 , and it becomes independent of temperature. These values are consistent with previous measurements at room temperature in vacuum [17].

The friction data for steel on PTFE is shown in figure 8. The Hertzian contact stress is approximately $2.64 \mathrm{MPa}$. Below $200 \mathrm{~K}$, there is essentially no temperature dependence of either $\mu_{\mathrm{k}}$ or $\mu_{\mathrm{s}}$. In contrast to the hard materials, the effects of wear appear to be minimal, and the friction coefficient measurements show no systematic variation with the number of sliding cycles. Between 250 and $450 \mathrm{~K}$, there is significant and repeatable temperature dependence which is hysteretic. In contrast again with the hard materials, the kinetic and static friction coefficients on PTFE show opposite behaviors: the static friction coefficient drops by nearly a factor of 5 from $\mu_{\mathrm{s}}=0.25$ at $\mathrm{T}=200 \mathrm{~K}$ to $\mu_{\mathrm{s}}=0.06$ at $\mathrm{T}=450 \mathrm{~K}$, while the kinetic friction has a feature at $T=370 \mathrm{~K}$ which is a maximum on warming and a minimum on cooling. The position of the maximum and minimum depend on the cooling and heating rate. The characteristic relaxation time for the transition from one state to another is approximately $3 \mathrm{~h}$. This hysteretic behavior may be due to a phase transition that is known to occur in PTFE in the vicinity of $300 \mathrm{~K}[18,19]$.

After the sliding measurements on each sample were completed, the track was examined for evidence of wear. Optical microphotographs are shown in figure 9. For the steel-PTFE system, no particles were observed, and there was no optically detectable transfer film on the steel ball, but the track in the PTFE was visible, primarily due to an increased reflectivity; the track had a smooth appearance with no apparent scratches. For the steel on steel system, we observed clearly visible wear tracks which roughened the mirror-like finish of the sliding surface. The track is composed of many small scratches parallel to the direction of motion which are approximately 300 microns in length. Both steel and sapphire balls on sapphire produced a visible track in the sapphire as well as numerous fine white particles which were presumably sapphire. The sapphire ball showed stress cracks and both the sapphire and steel balls were covered with clumps of the fine white particles.
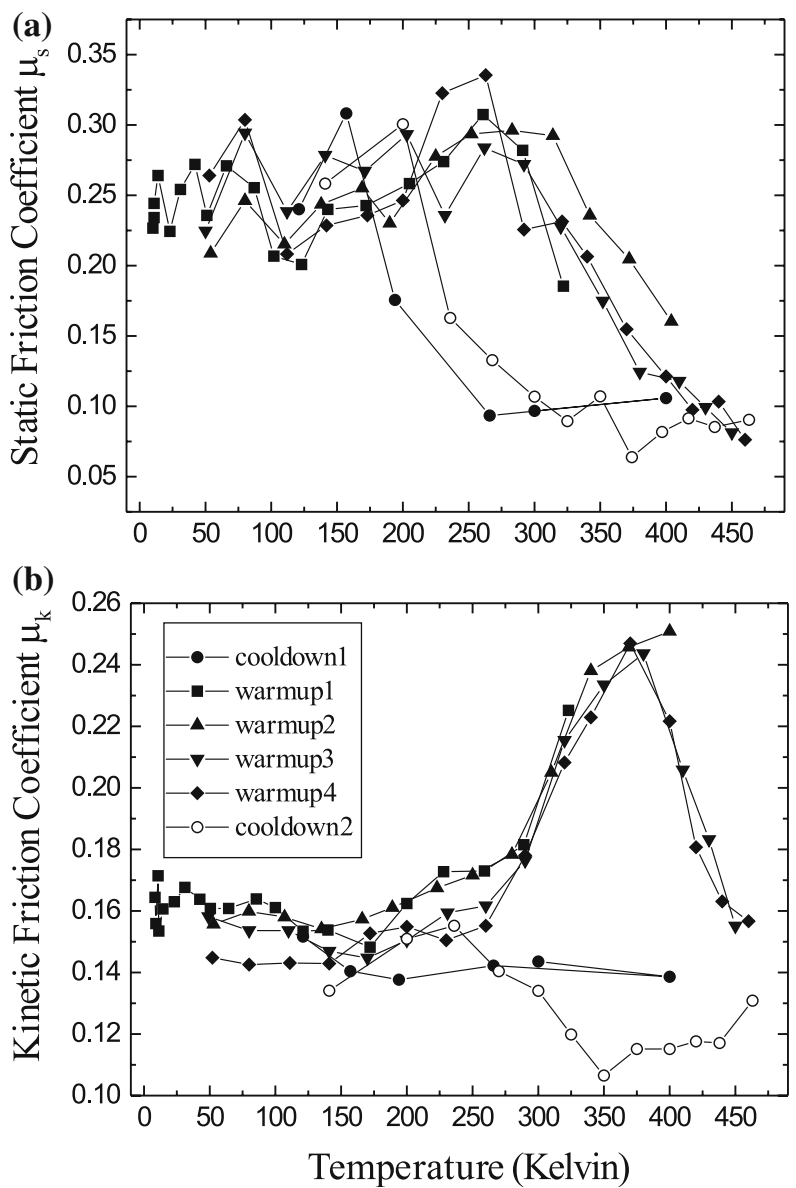

Figure 8. Friction coefficients of steel on PTFE (Teflon) coating as a function of temperature. The symbols indicate whether the data was taken on heating or cooling, and the line segments connecting the data points indicate the order in which they were measured. (a) static friction, (b) sliding friction. 

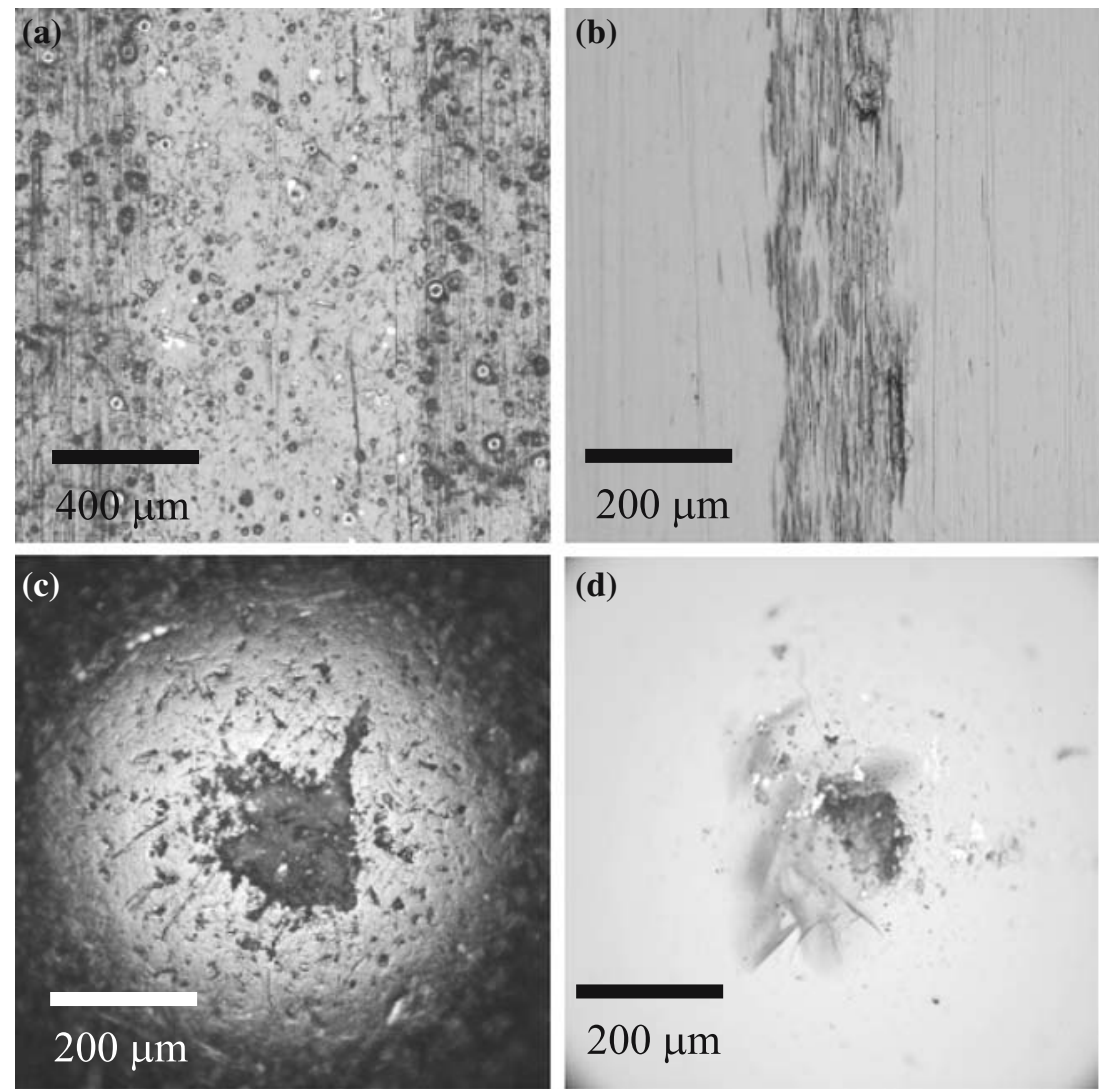

Figure 9. Microphotographs of wear. Bars indicate scale. (a) Wear track on PTFE coating created by steel ball. (b) Wear track of steel on steel. (c) Wear and debris on a steel ball after sliding on sapphire. (d) Wear cracks and debris on sapphire ball after sliding on a sapphire plate.

\section{Conclusion}

There are many theoretical reasons to expect a strong temperature dependence of the friction coefficient, particularly in the cryogenic regime. In contrast to these expectations, as well as some recent AFM work [11], we find essentially no temperature dependence to either the static or dynamic friction in the temperature range $4 K<T<200 K$ for all the systems we investigated. Despite the relatively low loads in our experiments, wear was found to be an important factor. For all the hard materials, the dynamic friction was initially low, and made an irreversible transition to a high friction state. In these materials, we believe that the effects of wear overwhelm any possible temperature dependent friction mechanisms. Our results show that PTFE coatings have many practical advantages for cryogenic engineering. Standard commercially available PTFE coatings on stainless steel can be thermally cycled to cryogenic temperatures many times and survive intact with no degradation of their properties. The low temperature dynamic friction of steel on PTFE was approximately 0.18 , which was less than one half that of the other materials we tested. Wear does not seem to be nearly as significant as in the hard materials, and the friction coefficient was a repeatable function of temperature, independent of the number of sliding cycles.

\section{Acknowledgments}

We thank J. Krim, K.J. Wahl and R.R. Chromik for useful discussions. This work has been supported by EXTREME FRICTION AFOSR MURI \# FA9550-04$1-0381$.

\section{References}

[1] V.L. Popov, Trib. Int. 34 (2001) 277.

[2] F.P. Bowden and D. Tabor, The friction and lubrication of solids (Claredon Press, 2001).

[3] M. Akbulut, A.R. Godfrey Alig and J. Israelachvilli, J. Chem. Phys. 124 (2006) 174703.

[4] T. Baumberger, P. Berthoud and C. Caroli, Phys. Rev. B 60 (1999) 3928.

[5] Y. Sang, M. Dube and M. Grant, Phys. Rev. Lett. 87 (2001) 174301.

[6] N.L. McCook, D.L. Burris, P.L. Dickrell and W.G. Sawyer, Trib. Lett. 20 (2005) 109.

[7] G. Theiler, W. Hubner, T. Gradt, P. Klein and K. Friedrich, Trib. Int. 35 (2002) 449.

[8] P.C. Michael, E. Rabinowicz and Y. Iwasa, Cryogenics 31 (1991) 695. 
[9] W. Hubner, T. Gradt, T. Schneider and H. Borner, Wear 216 (1998) 150.

[10] B. Subramonian and B. Basu, Mat. Sci. Eng. A 415 (2006) 72.

[11] A. Schirmeisen, L. Jansen, H. Holscher and H. Fuchs, Appl. Phys. Lett. 88 (2006) 123108.

[12] J.C. Burton, P. Taborek and J.E. Rutledge, J. Low Temp. Phys. 134 (2004) 237.

[13] T. McMillan, J.E. Rutledge and P. Taborek, Rev. Sci. Instrum. 75 (2004) 5005.
[14] T. McMillan, J.E. Rutledge and P. Taborek, J. Low temp. Phys. 138 (2005) 995.

[15] R. Chen, A. Iwabuchi and T. Shimizu, Wear 249 (2001) 379.

[16] A. Iwabuchi, S. Iida, Y. Yoshino, T. Shimizu, M. Sugimoto, H. Nakajima and K. Yoshida, Cryogenics 33 (1993) 1110.

[17] J.M. Gorges, D. Mazuyer, P. Kapsa and T. LeMogne, Trib. Trans. 35 (1992) 2.

[18] D.G. Flom and N.T. Porile, Nature 175 (1955) 682.

[19] R.I. Beecroft and C.A. Swenson, J. Appl. Phys. 30 (1959) 1793. 